\title{
Confusion and visual field effects in rhyme and name letter matching
}

\author{
DAVID B. BOLES \\ Rensselaer Polytechnic Institute, Troy, New York
}

\begin{abstract}
Recent evidence (Boles \& Eveland, 1983) suggests that mixed-case letter pairs such as $A a$ are matched not through a common phonetic or name code, but rather through the detection of visual equivalence between alternate letter cases. The generation model states that equivalence is detected by internally generating the opposite cases of the letters, which are then compared to the stimulus pair trace. What is lacking in this formulation, however, is evidence that the phonetic or name code can be used for other letter-matching tasks and that it has a predominantly lefthemisphere lateralization. Here eight experiments employed rhyme- and name-matching tasks along with manipulations of phonetic and generation confusion. Meta-analysis of the reaction time and error results shows the expected interaction of task and type of confusion. In addition, a small right visual field or left hemisphere advantage was found for rhyme matches, but not for name matches. It is concluded on both confusion and lateralization grounds that rhyme- and name-matching tasks employ different processes, and that these can be described, respectively, as phonetic and visual in nature.
\end{abstract}

Although it has usually been assumed that phonetic representations of letter names are used in the samedifferent matching of mixed-case letter pairs such as $A a$ or $A b$, accumulating evidence suggests that this is not in fact the case. Most notably, attempts to produce phonetic confusion by introducing noise letters (Posner \& Taylor, 1969 ) or by varying the phonetic confusability of different pair members (Boles \& Eveland, 1983) have not generally succeeded in showing an effect on the time taken to match letters or on errors made in letter matching (for a critical review of other literature, see Boles \& Eveland, 1983). In addition, a right visual field (left hemisphere) superiority often fails to appear in studies using lateralized presentation of letter pairs, although generally speaking, such a superiority is predicted when verbal processes are involved (Birkett, 1981; Boles, 1981; Hellige, 1975; Wilkins \& Stewart, 1974). Empirically, superiority of the left hemisphere is generally found for verbal processing (Beaumont, 1982b; Bryden, 1982), even though the right hemisphere is believed to have some linguistic competence (Beaumont, 1982a; Searleman, 1977).

Together, the lack of phonetic confusion and visual field effects for so-called name matches suggest that phonetic processes are not used. Yet if phonetic processes are not involved, how can subjects detect equivalence between two letters of different form? Recently, Boles and Eveland

Reprint requests should be addressed to David B. Boles, Department of Psychology, Rensselaer Polytechnic Institute, Troy, NY 12180. The author wishes to thank the Department of Psychology, University of Oregon, for making the facilities of the Cognitive Laboratory available for Experiments 1 and 2, with special thanks to Gil Osgood and Al Murphy for the design of the personal computer system software used in Experiments 3, 4, 6,7, and 8. Thanks also go to Van Catterall and Lori Cohan for running Experiment 8. Experiment 5 was conducted with support from Grant No. 17681 from the University of Calgary.
(1983) attempted to fill this theoretical gap by proposing a generation model of letter matching. Put in its most general terms, this model states that either or both members of a stimulus pair such as $A a$ generate a memory representation of the opposite case (i.e., the stimulus $A$ generates $a$, and/or the stimulus $a$ generates $A$ ). Each generated representation can then be visually matched to one or both of the original stimulus letters, in this instance supporting a same response (the generated $a$ matches the original $a$, and/or the generated $A$ matches the original $A$ ). In effect, the theoretical gap is filled (according to this proposal) by a rapid generation process in which generated memories possess visual characteristics and support visual matches for equivalence.

Evidence in support of the model comes from the manipulation of generation confusion. When Boles and Eveland (1983) constructed different pairs so that any generated letters would be visually confusable with the original letters (e.g., the pair $G q$ ), reaction times were 50-120 msec longer than when nonconfused pairs were used. A follow-up experiment demonstrated that the effect was not due to direct visual confusion between the stimulus letters themselves (e.g., between $G$ and $q$ ).

A useful addition to the results of Boles and Eveland (1983) would be a task in which phonetic processes must be employed. For instance, phonetic processes would be expected to be used in rhyme matches based on the terminal sounds of letters (e.g., $A k$ ). An experimental investigation might show that rhyme matching is influenced by phonetic confusion or visual field of presentation, and that it is insensitive to generation confusion. If so, then name matching would have been dissociated from rhyme matching, because name matches are insensitive to phonetic confusion and visual field of presentation but are highly sensitive to generation confusion. Such 
a pattern of dissociation from a phonetic task would support the thesis of Boles and Eveland, that is, that name matches are, in effect, visual and not phonetic matches.

The following experiments were designed to contrast name letter matching with rhyme matching, with an aim toward dissociating the two tasks with respect to (1) the type of effective confusion and (2) visual-field effects. The rhyme task required subjects to judge whether two letter names rhyme (e.g., $J k, C b)$ or not (e.g., $J b, C k$ ). It was predicted that this task, unlike the name-matching task, should result in greater phonetic than visual (generation) confusion, and in a right-field superiority, given lateralized presentation. By contrast, the name-matching task should result in relatively greater visual confusion and in an essentially null visual-field difference (Boles, 1981; Boles \& Eveland, 1983).

\section{METHOD}

\section{General Method}

In experiments using a name-matching task, letter pairs were constructed so that some pairs had the same name but were physically nonidentical (e.g., $A a$ ), whereas the remainder were different pairs (e.g., $A b$ ). Similarly, experiments using a rhyme-matching task used pairs whose terminal sounds were the same (e.g., $A k$ ), or not (e.g., $A b$ ).

Several experiments also varied the type of confusion present in different pairs. Typically, in both the rhyme- and name-matching tasks, phonetically confused pairs were defined as nonrhyming pairs showing confusability in Conrad's (1964) acoustic confusion matrix (e.g., Hs). Generation-confused pairs were those in which the opposite case of each letter physically resembled the other letter according to the visual confusion matrices of Hodge (1962) (e.g., $Q g$ ) Importantly, in all experiments using both types of letter matching, the different pairs were the same regardless of the task.

Experiments 2, 3, 4, and 6 used the same stimulus set (idiosyncratic sets were used in Experiments 1, 5, 7, and 8). Because the set was modal, it is described in detail here. Same pairs for the rhyme-matching task were $B p, C g, G c, G t, J k, K j, P b, Q u, T g$, and $U q$. For the name-matching task, the same pairs were $F f, G g$, $H h, K k, N n, O o, Q q, T t, U u$, and $X x$. For both tasks, phonetically confused different pairs were $G u, H s, F x, K n, N k, Q t, S h, T q, U g$, and $X f$; generation-confused different pairs (Boles \& Eveland, 1983) were $F t, G o, G q, H n, K x, N h, O g, Q g, T f$, and $X k$. As calculated from the confusion matrices of Conrad (1964) and Hodge (1962), mean phonetic confusion was $130.8(S D=65.7)$ for the phoneticconfusion pairs and $25.7(S D=26.5$ ) for the generation-confusion pairs. Mean generation confusion was $0.3(S D=0.4)$ and 19.7 $(S D=11.1)$ for these sets, respectively. ${ }^{1}$

A computer (a PDP-15, Apple II+, or Apple Ile, depending on the experiment) presented the pairs and accepted manual keypresses from the subjects. Trial time-lines varied across experiments, but typically went as follows: (1) presentation of a fixation cross, followed by (2) the stimulus display for some set duration, always 150 msec or less; (3) the subject's response or a set deadline; and (4) brief feedback about reaction time (RT) and the correctness of the response. Responses were made on keys arranged in the midline, to reduce compatibility effects. Stimulus displays always involved lateralized presentation, with one or both members of the pair presented in either the left visual field (LVF) or right visual field (RVF). Also, a small amount of practice was given before data were collected for each task, and the order of task presentation was balanced across subjects. All subjects were right-handed by self-report.

\section{Specific Methods}

Because the results of the eight experiments are presented in terms of meta-analyses, a detailed treatment of specific methods is not warranted. Accordingly, each experiment is briefly described in terms of its unusual features or departures from the general method. Further details are available from the author on request. Generally speaking, the variations of method across experiments were due to a desire to identify the optimal methods for establishing confusion and visual-field effects. It is clear, however, that no one method proved optimal in these respects, although the results of all experiments combined do produce a reasonably coherent picture.

Experiment 1. A single lowercase letter was presented at fixation, with an uppercase letter presented peripherally and flanked (at the top and bottom) by two noise letters. The match was to be made between the letter at fixation and the flanked peripheral letter. When the letters to be matched were different, the flanking letters showed either generation or phonetic confusion with the central letter, and so defined the generation-confused and phonetically confused conditions. For example, when the central letter $k$ was to be matched to the peripheral letter $D$, the flanking letters $J$ and $A$ operationalized phonetic confusion, whereas generation confusion was operationalized by $R$ and $X$. There were 48 stimulus displays. Both name and rhyme matches were used. The 16 subjects were from the University of Oregon Cognitive Laboratory subject pool; each contributed a total of 576 trials.

Experiment 2. Both letters to be matched were presented peripherally, stacked vertically. No flanking letters were used, so that confusion was defined within the pair, as described for the modal stimulus set in the General Method section. Both name and rhyme matches were employed. The 12 subjects, from the University of Oregon Cognitive Laboratory subject pool, contributed 720 trials each.

Experiment 3. One letter was presented at fixation, with the other presented peripherally. Both name and rhyme matches were used. The 16 subjects, from Ilinois State University, contributed 600 trials each.

Experiment 4. Successive letter matches were used, the two letters separated by a stimulus onset asynchrony of $2,400 \mathrm{msec}$, with the fixation cross instated for most of the intervening period. Otherwise the experiment was similar to Experiment 3 , including the number and source of subjects and trials.

Experiment 5. Pairs were simultaneous, with one letter at fixation and one in the periphery. Although rhyme matching was used as one task, the control task was physical matching, not name matching. Same pairs for physical matches were visually identical (e.g., $A A)$; different pairs were not (e.g., $A P$ ). All letters in both tasks were uppercase. No confusion conditions were used, so this experiment primarily contributed data on visual-field effects. The 29 subjects were from the University of Calgary; each subject contributed 192 trials.

Experiment 6. Only rhyme matching was used. Letters were always uppercase, and pairs were presented in three different ways: with one letter at fixation and the other shown peripherally; with both letters shown peripherally, stacked vertically; and with both letters shown peripherally, but with a digit at fixation. For the third type of display, subjects made a second response to classify the digit as odd or even. Inclusion of this condition was motivated by the previous finding (Boles, 1983) that under some circumstances, a central digit magnifies obtained visual-field asymmetry. No confusion conditions were used in the experiment. The 12 subjects, from the University of Illinois at Urbana-Champaign, contributed 720 trials each.

Experiment 7. Only rhyme matching was used. Letters were uppercase, and either two or three letters were presented. If two letters were shown, either both were presented peripherally or one was presented centrally and the other peripherally. If three letters were shown, either all three were presented peripherally or one was presented centrally and two peripherally. All letters had to rhyme 
for a same response. There were no confusion conditions. The 16 subjects, from the University of Illinois at Urbana-Champaign, contributed 512 trials each.

Experiment 8. Both name and rhyme matches were employed. A mixed-case pair was presented peripherally, arranged horizontally, with an arrow at fixation pointing to the pair. A randomly selected distractor pair, shown in the other visual field, was to be ignored; this manipulation was motivated by the finding of Boles (1985) that the presence of a distractor stimulus in the opposite visual field often increases the size of reaction time asymmetries. The 12 subjects, from Rensselaer Polytechnic Institute, contributed 480 trials each.

\section{RESULTS}

In general, median RTs and percent errors were calculated for each condition of an experiment, and analyses of variance were conducted to assess the significance of relevant effects. Results were then combined across experiments using the Stouffer method of meta-analysis (Rosenthal, 1978). In this method, individual one-tailed probability levels are converted to $z$ scores, the $z$ scores are summed across experiments, and the sum is divided by the square root of the number of experiments. The result is an overall $z$ score which will here be termed $z^{\prime}$, which is then converted to an overall one-tailed probability level $\left(p^{\prime}\right)$.

In addition, the homogeneity of the results of each metaanalysis was tested using the chi-square method given by Rosenthal and Rubin (1979). With one exception, which is noted, all tests were passed, so the results of the individual experiments appear to be comparable. It should be emphasized that the experiments included in the metaanalysis are all of those completed at this writing, so they do not represent a biased selection.

\section{Confusion Effects}

Table 1 shows the task $\times$ confusion interaction in different pair RT for each of the experiments in which rhyme matches were contrasted with an appropriate control conditions and confusion conditions were present (Experiments 1-4). The interaction proved highly robust, appearing in each experiment individually, and it was highly significant by meta-analysis $\left(z^{\prime}=4.31, p^{\prime}=.000008\right)$. In Experiment 1, which was unique in that virtually identical different pairs were used for the phonetic-confusion and generation-confusion conditions (the irrelevant flank-

Table 1

Task $\times$ Confusion: RT (in Milliseconds) for Different Pairs

\begin{tabular}{|c|c|c|c|c|c|c|c|}
\hline \multirow[b]{2}{*}{ Experiment } & \multicolumn{2}{|c|}{ Rhyme } & \multicolumn{2}{|c|}{ Name } & \multirow[b]{2}{*}{ Interaction } & \multirow[b]{2}{*}{$p$} & \multirow[b]{2}{*}{$Z$} \\
\hline & PHO & $\overline{\text { GEN }}$ & $\overline{\mathrm{PHO}}$ & $\overline{\text { GEN }}$ & & & \\
\hline 1 & 1,080 & 1,052 & 754 & 764 & +38 & .018 & +2.10 \\
\hline 2 & 698 & 752 & 670 & 767 & +43 & .015 & +2.17 \\
\hline 3 & 642 & 675 & 549 & 620 & +38 & .013 & +2.23 \\
\hline 4 & 618 & 635 & 514 & 561 & +30 & .017 & +2.12 \\
\hline Mean & 760 & 779 & 622 & 678 & +37 & $\begin{array}{r}z^{\prime}= \\
p^{\prime}=\end{array}$ & $\begin{array}{c}+4.31 \\
.000008\end{array}$ \\
\hline
\end{tabular}

Note $-P H O=$ phonetic confusion, $G E N=$ generation confusion.
Table 2

Task $\times$ Confusion: Percent Errors for Different Pairs Rhyme Name

\begin{tabular}{ccccccccc} 
Experiment & PHO GEN & & \multicolumn{2}{c}{ PHO GEN } & & & & \\
\hline 1 & 6.0 & 6.4 & 3.1 & 3.2 & -.3 & .649 & -.38
\end{tabular}

$\begin{array}{rrrrrrcr}1 & 6.0 & 6.4 & 3.1 & 3.2 & -.3 & .649 & -.38 \\ 2 & 12.4 & 9.3 & 4.9 & 21.5 & +19.7 & <.00001 & >+4.26\end{array}$

$\begin{array}{lllllll}3 & 10.4 & 7.7 & 2.7 & 11.2 & +11.2<.000005>+4.42\end{array}$

$\begin{array}{llllllll}4 & 8.9 & 6.8 & 3.2 & 6.2 & +5.1 & .0002 & +3.53\end{array}$

$\begin{array}{lllllll}\text { Mean } & 9.4 & 7.6 & 3.5 & 10.5 & +8.8 & z^{\prime}>+5.92\end{array}$

$p^{\prime}<2 \times 10^{-\rightarrow}$

Note $-P H O=$ phonetic confusion, $G E N=$ generation confusion .

Table 3

\begin{tabular}{|c|c|c|c|c|c|c|c|}
\hline \multicolumn{8}{|c|}{ Visual Field $\times$ Task: RT (in Milliseconds) } \\
\hline \multirow[b]{2}{*}{ Experiment } & \multicolumn{2}{|c|}{ Rhyme } & \multicolumn{2}{|c|}{ Control } & \multirow[b]{2}{*}{ Interaction } & \multirow[b]{2}{*}{$p$} & \multirow[b]{2}{*}{$Z$} \\
\hline & LVF & RVF & LVF & RVF & & & \\
\hline 1 & 1,081 & 1,049 & 768 & 758 & +22 & .065 & +1.51 \\
\hline 2 & 735 & 741 & 718 & 733 & +10 & .096 & +1.30 \\
\hline 3 & 678 & 670 & 584 & 586 & +10 & .158 & +1.00 \\
\hline 4 & 631 & 628 & 532 & 530 & +1 & .460 & +.10 \\
\hline 5 & 895 & 885 & 645 & 640 & +5 & .387 & +.29 \\
\hline \multirow[t]{2}{*}{8} & 980 & 974 & 860 & 895 & +41 & .009 & +2.75 \\
\hline & & & & & & $\begin{array}{l}z^{\prime}= \\
p^{\prime}=\end{array}$ & $\begin{array}{c}+2.84 \\
.003\end{array}$ \\
\hline
\end{tabular}

Note $-L V F=$ left visual field, $R V F=$ right visual field .

ing letters were varied), the interaction showed a crossover form in that for rhyme matches, phonetically confused pairs produced longer RTs than generationally confused pairs, whereas the opposite was true of name matches. In Experiments 2-4, all of which used the same letter pairs in which type of confusion was more directly implemented (within a pair, rather than via flanking letters), the interaction took the form of a reduced difference (generation confusion minus phonetic confusion) for rhyme matches, compared with that found for name matches. A possible reason for the differing forms of the interaction is discussed below.

The percent error effects are shown in Table 2. The meta-analysis again revealed a highly significant task $x$ confusion interaction $\left(z^{\prime}>5.92, p^{\prime}<2 \times 10^{-9}\right)$. Furthermore, here the overall interaction has a crossover form, with more errors made for phonetically confused than for generationally confused pairs in the rhyme task, and vice versa in the name task. However, the test for homogeneity was not passed in this instance $\left[\chi^{2}(3)=\right.$ $15.30, p<.01]$; as Table 2 clearly shows, the interaction was obtained only in Experiments 2-4.

\section{Visual-Field Effects}

Table 3 shows the visual field $\times$ task interactions in RT for all trials in those experiments that contrasted rhyme matching with a control task (Experiments 1-5 and 8). Although it was individually significant only in Experiment 8 , an interaction was obtained over experiments $\left(z^{\prime}\right.$ $=2.84, p^{\prime}=.003$ ).

Tables 4 and 5 examine the rhyme-matching and namematching RTs individually. The rhyme-matching results 
Table 4

Rhyme Matches: RT (in Milliseconds)

\begin{tabular}{crrrrr}
\hline Experiment & LVF & RVF & Difference & $p$ & \multicolumn{1}{c}{$z$} \\
\hline 1 & 1,081 & 1,049 & +32 & .009 & +2.35 \\
2 & 735 & 741 & -6 & .706 & -.54 \\
3 & 678 & 670 & +8 & .192 & +.87 \\
4 & 631 & 628 & +3 & .360 & +.36 \\
5 & 895 & 885 & +10 & .243 & +.70 \\
6 & 679 & 674 & +5 & .138 & +1.09 \\
7 & 784 & 781 & +3 & .284 & +.57 \\
8 & 980 & 974 & +6 & .394 & +.27 \\
Mean & 808 & 800 & +8 & $z^{\prime}=+2.00$ \\
& & & & $p^{\prime}=.023$ \\
\hline
\end{tabular}

Note $-L V F=$ left visual field, $R V F=$ right visual field .

Table 5

Name Matches: RT (in Milliseconds)

\begin{tabular}{cccccc}
\hline Experiment & LVF & RVF & Difference & $p$ & $Z$ \\
\hline 1 & 768 & 758 & +10 & .100 & +1.28 \\
2 & 718 & 733 & -15 & .898 & -1.27 \\
3 & 584 & 586 & -2 & .630 & -.33 \\
4 & 532 & 530 & +2 & .361 & +.36 \\
8 & 860 & 895 & -35 & .939 & -1.94 \\
Mean & 692 & 700 & -8 & $z^{\prime}=-.85$ \\
& & & & $p^{\prime}=.198$
\end{tabular}

Note $-L V F=$ left visual field, $R V F=$ right visual field .

include all experiments using this task (Experiments 1-8); overall, RVF pairs were responded to $8 \mathrm{msec}$ faster than LVF pairs ( 800 vs. $808 \mathrm{msec} ; z^{\prime}=2.00, p=.023$ ). By contrast, name matching (Experiments 1-4 and 8) showed no significant difference between the RVF and the LVF ( 700 vs. $692 \mathrm{msec} ; z^{\prime}=-.85$ ).

No visual field effects appeared in meta-analyses of the percent error data, either for the interaction or for the rhyme and name matches individually (all $z^{\prime} \mathrm{s}<1$ ).

\section{DISCUSSION}

The results of the eight experiments generally support the two hypotheses stated in the introduction. A dissociation was observed between rhyme- and name-matching tasks in the generation-confusion-phonetic-confusion difference, both in RT and in errors. In addition, a visualfield dissociation was observed: rhyme matches produced a small but significant RVF superiority in RT, whereas name matches resulted in a statistically null visual-field difference.

There are, however, some anomalies in the data which require explanation. The first is the varying form taken by the task $\times$ confusion interaction in RT between Experiment 1 and Experiments 2-4 (Table 1). Why was a crossover interaction found in Experiment 1, when a reduced difference interaction was found in the later experiments?

One possibility is that the kind of phonetic confusion employed in Experiments 2-4 was less effective than that used in Experiment 1. Because the confusion in Experi- ments 2-4 was implemented by the use of acoustic confusion matrices but was not based on the end sounds of letter names, subjects may have been able to ignore it because it was unrelated to the rhyming task. The result was relatively rapid RTs. By contrast, the flanking-letter confusion employed in Experiment 1 was based on end sounds, and may therefore have been more effective in disrupting rhyming matches.

Note, however, that a second anomaly in the results was a speed-accuracy tradeoff present for the rhyme-matching confusion conditions in Experiments 2-4 (Tables 1 and 2). Generationally confused pairs produced longer RTs, but fewer errors. This is perhaps explainable as a criterion effect traceable to the greater visual confusion in these pairs; that is, in perceiving greater visual similarity in these pairs, subjects may have responded with caution, producing longer RTs but greater accuracy. As a criterion effect, this would represent only a bias and not an effect on the ability to make rhyme matches per se.

According to this view, then, what was measured in Experiments 2-4 was the relative effect of generation (visual) confusion on rhyme and name matching, with non-end-sound phonetic confusion having no effect on either. Because generation confusion was direct, rather than indirect, its effect on name matches was greater in Experiments 2-4 than in Experiment 1.

In any case, the following conclusions seem reasonable. First, rhyme and name matches are probably not based on the same process, because a task $x$ confusion interaction was consistently observed. ${ }^{2}$ Second, the nature of the interaction accords well with the interpretation that name matches rely much more heavily on visual processes than do rhyme matches.

The visual-field results provide additional support for these conclusions, because they imply another (hemispheric) dissociation of the two tasks. Specifically, rhyme matches produced a small but significant advantage of RVF over LVF presentations, whereas no asymmetry was observed for name matches. Ideally, of course, these results would have shown more reproducibility within single experiments than was obtained, so they do leave something to be desired. However, the direction of the visual field $\times$ task interaction (Table 3 ) was the same across six experiments, showing that the result, although small in size, is stable in direction. Furthermore, the direction of the visual-field difference for rhyme matches (Table 4) favored the RVF in seven of eight experiments. Finally, it should be noted that the obtained 8-msec RVF advantage is not far off the estimated lower boundary of visual interhemispheric transmission time (Bashore, 1981; Milner \& Lines, 1982).

The significant result of the visual field $\times$ task RT metaanalysis implies that different neurological substrates underlie the two tasks: rhyme matches apparently rely more on left hemisphere than on right hemisphere processes, whereas name matches seem to be within the equivalent competencies of both hemispheres. The former finding 
would be expected from the phonetic nature of rhyme matches (Zaidel \& Peters, 1981), whereas the latter shows striking similarity to previous results (see Boles, 1981, for a review and original data).

Thus, in large part, the results seem to satisfy the major goals outlined in the introduction. When a letter-matching task is used which requires phonetic processing (rhyme matching), the outcome is (1) a reduction in the effect of visual (generation) confusion, compared to name matching, and (2) a RVF advantage in reaction time. These observations agree with the preponderant lateralization of language to the left hemisphere of right-handed people, and, inasmuch as no field effect was found for name matches, they indicate that name matches do not rely on language processes in the same sense as rhyme matches. The large effects of generation confusion obtained for name matches further support the conclusion of Boles and Eveland (1983) that visual memory processes underlie the matching of name-identity pairs. Indeed, it seems that the term name match is a misnomer, applying only to a formal relationship between alternative cases and not to the mental processes actually used by subjects in judging letter equivalence.

Incidentally, the present experiments provide evidence against a competing interpretation of the generationconfusion results of Boles and Eveland (1983). An anonymous reviewer has suggested that the effect of generation confusion on name matches may be a disguised effect of phonetic confusion. That is, a generation-confused pair such as $Q g$ may activate the visually similar letters $G$ and $q$ (which is also predicted by the generation model of Boles and Eveland), and phonetic (name) confusion may therefore exist because of the name equivalencies between $G$ and $g$ and between $Q$ and $q$ (which is not predicted by the generation model). However, such an interpretation encounters difficulty in explaining the lesser effect for rhyme matches obtained in the present experiments. The letters $G$ and $g$ certainly end in the same sound, as do the letters $Q$ and $q$, so one might expect similar effects of generation confusion on rhyme matches. Instead, a reduced effect was found. Furthermore, hypothesis of disguised phonetic confusion runs into difficulties on other grounds, including (1) the absence of a visualfield effect for name matches, which might be expected if the matches are ultimately based on phonetic processes, and (2) the lack of a phonetic-confusion effect for name matches (the null effect has been established repeatedly by Boles and Eveland, 1983). Of course, one can never rule out the possibility of ancillary assumptions that can be added to the phonetic explanation to explain the full complex of results. Yet it seems likely that any such set of assumptions would be complex compared to a nonphonetic view of name matching, and would suffer from a cut by Occam's razor.

Finally, one implication of these findings is that phonetic representations of letter names cannot be used as efficiently as visual representations of letter shapes in matching for name identity, at least when presentations are visual. Otherwise, one would expect subjects to use phonetic processes, and some evidence of this would be found in the confusion or visual-field results. Congruent with this implication is the finding that rhyme matches averaged about $120 \mathrm{msec}$ longer than name matches (see Table 1).

\section{REFERENCES}

BASHORE, T. R. (1981). Vocal and manual reaction time estimates of interhemispheric transmission time. Psychological Bulletin, 89, 352-368.

BEAUMONT, J. G. (1982a). The split-brain studies. In J. G. Beaumont (Ed.), Divided visual field studies of cerebral organization. London: Academic Press.

Beaumont, J. G. (1982b). Studies with verbal stimuli. In J. G. Beaumont (Ed.), Divided visual field studies of cerebral organization. London: Academic Press.

BirkeTt, P. (1981). Hemispheric asymmetry for classifying upright and inverted letter pairs: Handedness and sex differences. Neuropsychologia, 19, 713-717.

Boles, D. B. (1981). Variability in letter-matching asymmetry. Perception \& Psychophysics, 29, 285-288.

BoLEs, D. B. (1983). Hemispheric interaction in visual field asymmetry. Cortex, 19, 99-113.

Boles, D. B. (1985, November). Bilateral vs. unilateral stimulation, and reaction time asymmetries. Paper presented to the Annual Meeting of the Psychonomic Society, Boston.

Boles, D. B., \& Eveland, D. C. (1983). Visual and phonetic codes and the process of generation in letter matching. Journal of Experimental Psychology: Human Perception \& Performance, 9, 657-674.

BRYDEN, M. P. (1982). Laterality: Functional asymmetry in the intact brain. New York: Academic Press.

Conrad, R. (1964). Acoustic confusions in immediate memory. British Journal of Psychology, 55, 75-84.

Hellige, J. B. (1975). Hemispheric processing differences revealed by differential conditioning and reaction time performance. Journal of Experimental Psychology, 104, 309-326.

HodGE, D. C. (1962). Legibility of a uniform-strokewidth alphabet: I. Relative legibility of upper and lower case letters. Journal of Engineering Psychology, 1, 34-46.

Milner, A. D., \& LiNes, C. R. (1982). Interhemispheric pathways in simple reaction time to lateralized light flash. Neuropsychologia, 20, 171-179.

Posner, M. I., \& TAYlor, R. L. (1969). Subtractive method applied to separation of visual and name components of multiletter arrays. Acta Psychologica, 30, 104-114.

Rosenthal, R. (1978). Combining results of independent studies. Psychological Bulletin, 85, 185-193.

Rosenthal, R., \& RuBIN, D. B. (1979). Comparing significance levels of independent studies. Psychological Bulletin, 86, 1165-1168.

SEARleman, A. (1977). A review of right hemisphere linguistic abilities. Psychological Bulletin, 84, 503-528.

Wilkins, A., \& STEWART, A. (1974). The time course of lateral asymmetries in visual perception of letters. Joumal of Experimental Psychology, 102, 905-908.

Zaidel, E., \& Peters, A. M. (1981). Phonological encoding and ideographic reading by the disconnected right hemisphere: Two case studies. Brain \& Language, 14, 205-234.

\section{NOTES}

1.Confusion indices were calculated as in the following examples. For the phonetically confused pair $H s$, the mean number of acoustic confusions was extracted from Conrad's (1964) matrix as the number 
of $H$ responses to an $S$ auditory stimulus (43), plus the number of $S$ responses to an $H$ stimulus (151), divided by $2(97)$. Thus, the phoneticconfusion index for $H s$ was taken to be 97 , and the mean phonetic confusion (and $S D$ ) was derived by averaging the indices for all stimulus pairs within a set. Similarly, for the generation-confused pair $H n$, the mean number of cross-case visual confusions was extracted from Hodge's (1962) matrices as the number of $H$ responses to an $N$ visual stimulus (7), plus the number of $N$ responses to $H(71)$, plus the number of $h$ responses to $n(4)$, plus the number of $n$ responses to $h$ (3), divided by $4(21.25)$. The mean generation confusion (and $S D$ ) was then derived across all stimulus pairs within a set.
2. Robert Proctor has suggested that the apparent effects of confusion in Experiment 1 were due to the noise letters' contributing information toward a same response, an effect that would take place regardless of the particular code subjects used to match rhyming letters. The point is well taken and does constitute a plausible alternative explanation for the confusion results in Experiment 1.

(Manuscript received April 30, 1984; revision accepted for publication February 6, 1986.) 